Ann. Zootech., I973, 22 (3), 303-310.

\title{
PERFORMANCES DES BREBIS ROMANOV SOUMISES A UN RYTHME ACCÉLÉRÉ DE REPRODUCTION
}

\author{
L. TCHAMitChian, G. RICORDEAU. C. LEFÈVRE et A. DESVIGNES \\ avec la collaboration technique de $\mathrm{D}$. Lajous \\ Laboratoire de Génétique des Petits Ruminants, \\ Centre de Recherches de Toulouse, I. N.R. A.. \\ B. P. 12, 31320 Castanet Tolosan
}

\section{RÉSUMÉ}

Le système de 4 luttes en 2 ans (lutte I en mai-juin 1969, lutte 2 de septembre à novembre I969, lutte 3 en mai-juin I970, et Iutte 4 en octobre I970) a été appliqué à 66 brebis adultes Romanov (groupe I) dont 55 avaient mis bas 3 à 4 mois avant la lutte I (tabl. r).

Le taux de fertilité global aux luttes I, 2, 3 et 4 est respectivement de 75,8; 65, I; 7,o et 87,7 p. Ioo; les taux de prolificité correspondants sont de $256,293,225$ et 340 p. roo (tabl. 3 ). Lorsqu'on considère uniquement les brebis gravides à la lutte précédente, le taux de fertilité est de 87,3 à la lutte $I$, de 55,3 à la lutte 2 et de o aux luttes 3 et 4 (tabl. I).

Les résultats obtenus sur 3 I agnelles (groupe II), saillies pour la première fois à la lutte 2 de septembre-octobre 1969 , confirment les mauvais résultats de la lutte 3 de mai I970 (tabl. I).

Pour obtenir une meilleure productivité en rythme accéléré, il semble nécessaire de modifier les périodes de lutte pour mieux tenir compte de l'activité sexuelle des brebis Romanov en fonction de la saison.

\section{INTRODUCTION}

Dans leurs études consacrées à la race Romanov (cf. revue bibliographique de DESVIGNES, I97I), les auteurs russes indiquent que les brebis de cette race présentent la particularité de n'avoir pratiquement pas d'anœstrus saisonnier et de pouvoir être fécondées 30 à 40 jours après la mise bas, ce qui rend théoriquement possible deux agnelages par an. Nous avons voulu étudier cette possibilité sur le troupeau Romanov du domaine de I,a Sapinière, près de Bourges, en pratiquant 2 luttes à période fixe : 
octobre-novembre et mai-juin. Ces deux périodes ont été choisies en 1969 , car à l'époque, compte tenu des observations de ThIMonier et MaUlÉon (I969) sur brebis Ile-de-France et Préalpes $d u s u d$, nous espérions bénéficier, en plus de l'activité sexuelle maximum d'octobre-novembre, d'une activité sexuelle secondaire en maijuin. Nous rapportons ici les résultats obtenus.

\section{CONDITIONS EXPÉRIMENTALES}

\section{A. - Animaux}

Le système des 2 luttes annuelles a été appliqué de i969 à 197 I à 2 groupes de brebis.

Groupe 1: 66 brebis au total. En mai 1969 , lors de la lutte $\mathrm{I}$, ce groupe comprend :

- 55 brebis ayant mis bas du 25-I 2-I 968 au 15-2-1969, dont 42 multipares et I 3 primipares ;

- II brebis mises hors reproduction du I-8-1967 au 30-3-69 pour l'étude des variations saisonnières du comportement d'œstrus cyclique, dont 7 nullipares âgées de 28 mois et 4 brebis âgées de 4 à 5 ans ayant fait au moins une gestation.

Pendant la période considérée, 9 brebis ont été réformées ( 3 après la lutte I et 6 après la Iutte 2) pour des raisons sanitaires indépendantes des performances de reproduction.

Groupe 2:31 agnelles nées entre le I9-12-I968 et le I6-I-I969. c'est-à-dire âgées de 8 à 9 mois à leur première lutte de septembre t969, début des observations.

\section{B. - Conditions de lutte}

Les dates des 4 périodes de lutte sont indiquées dans le tableau I, avec les dates de mises bas précédentes.

- lutte I et $3:$ lutte en lot, avec introduction des béliers le I ${ }^{\text {er }}$ mai,

- lutte 2 et 4 : lutte en main avec contrôle des chaleurs.

Après les mises bas de fin I968 début 1969, du groupe I, les mères ont allaité leurs agneaux pendant 2 à 3 mois, la séparation des agneaux étant intervenue au plus tard 15 jours avant le début de la lutte I. Après toutes les autres mises bas, les agneaux ont été séparés des mères 2 jours après la naissance et soumis à l'allaitement artificiel.

\section{C. - Conduite du troupeau}

A Bourges, le troupeau est habituellement soumis à une seule lutte par an en juillet-août. Les brebis vivent à l'extérieur sur pâturage à partir du mois d'avril et rentrent en bergerie, à la fin de novembre, pour l'agnelage et la période d'allaitement. Les brebis Romanov soumises aux deux luttes annuelles sont conduites un peu différemment : en mai, elles sont maintenues en bergerie pour y demeurer en présence des béliers, par lots séparés pendant toute la durée de la lutte ; elles sortent ensuite au pâturage et rentrent à nouveau en bergerie au fur et à mesure des mises bas, pour permettre leur tarissement et le déroulement de la lutte d'automne. Dans le cas de lutte en lot, on utilise de l'ordre de I mâle pour 6 femelles.

\section{D. - Définition des taux}

- Fertilité : brebis mettant bas en p. Ioo des brebis mises en lutte.

- Mise bas : brebis mettant bas en p. roo des brebis saillies.

- Prolificité : agneaux nés pour Ioo brebis agnelant,

-_ Fécondité : agneaux nés pour roo brebis mises en lutte. 
RYTHME DE REPRODUCTION DES BREBIS “ ROMANOV "

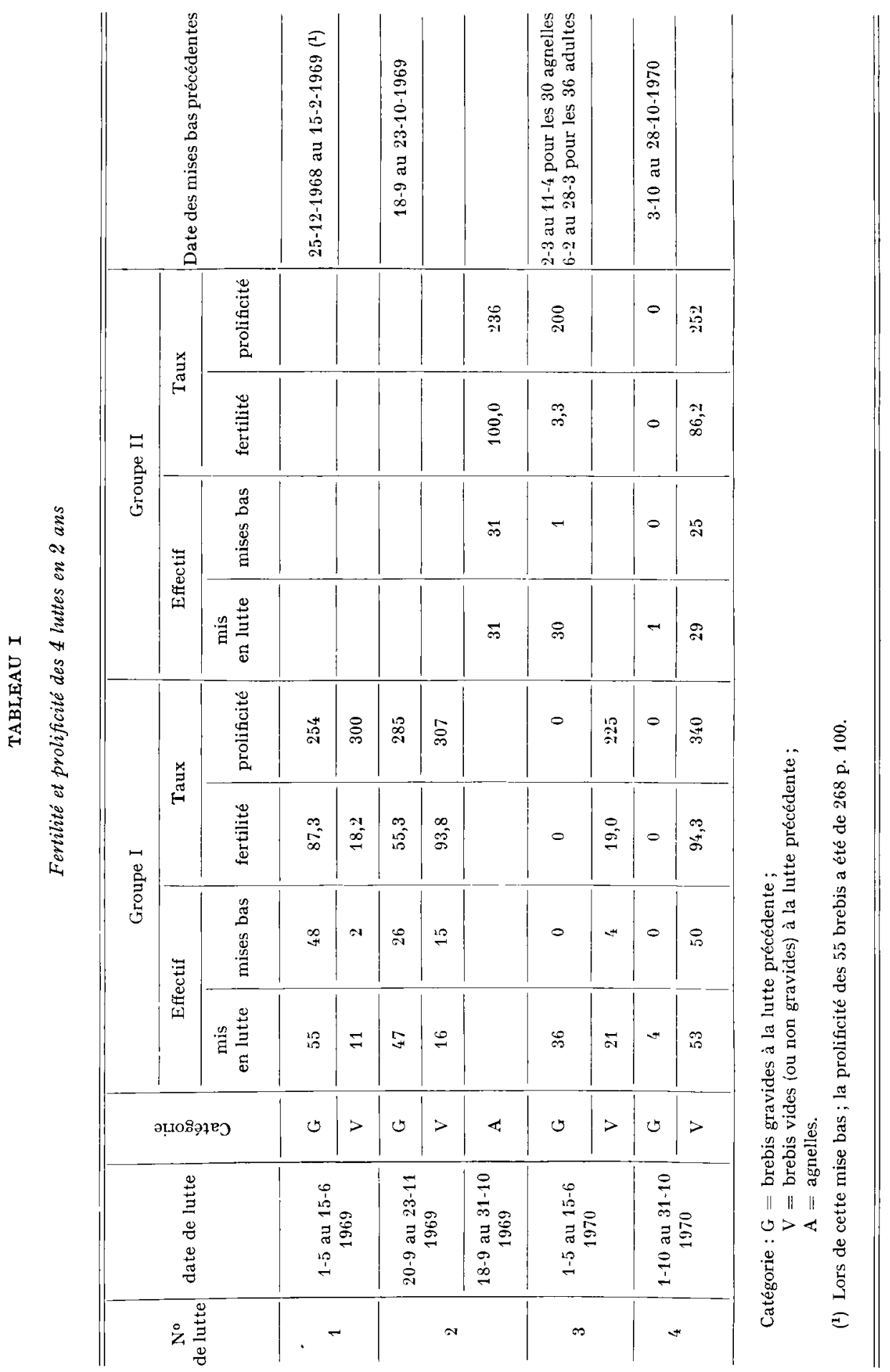




\section{RÉSULTATS}

\section{A. - Fertilité aux 4 luttes en fonction}

de la date de mise bas précédente (tabl. I)

Groupe 1 : à la première lutte de mai-juin I 969 , 1a fertilité est de 87,3 p. Ioo pour 55 brebis qui ont toutes mis bas du 25 décembre I968 au I5 février I969 et de I 8,2 p. Ioo seulement pour les brebis mises hors reproduction depuis 20 mois. La deuxième lutte a lieu en octobre-novembre, c'est-à-dire en saison sexuelle : sur les I 6 brebis précédemment vides, la fertilité est de 93,8 p. Ioo alors qu'elle est seulement de $55,3 \mathrm{p}$. Ioo pour les 47 brebis gravides à la lutte précédente. La troisième lutte de mai-juin est un échec, puisque seules 4 brebis précédemment vides ont été fécondées. Lors de la quatrième lutte d'octobre I970, la fertilité est de 94,3 p. Ioo sur les 53 brebis précédemment vides, mais aucune des 4 brebis ayant mis bas précédemment n'a été gravide à nouveau.

L'analyse des fécondations explique en partie les différences observées. L'enregistrement des œstrus n'a pas été fait lors de la lutte I de mai I969, cependant, la distribution des saillies fécondantes établie d'après les dates de mises bas fait apparaître deux séries de fécondations, les unes moins de 6 jours après 1'introduction du bélier, les autres $I_{5}$ à 27 jours après, ce qui permet de penser qu'il a pu y avoir un certain effet bélier à cette époque (fig. I).

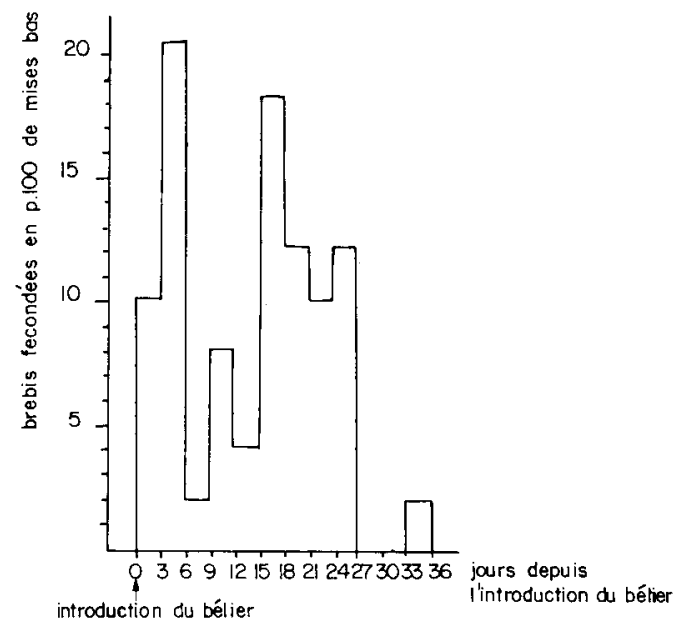

FIG. I. - Distribution des saillies fécondantes de la lutte 1

La date de saillie fécondante a été estimée d'après la date de mise-bas, compte tenu d'une gestation de I 42 jours

Lors des luttes 2 et 4 , les brebis précédemment vides sont fécondées rapidement: 83 et 87 p. Ioo après le premier œstrus ; 93 p. Ioo après les 2 premiers (tabl. 2). In revanche, lors de la lutte 2 , le taux de mise bas des brebis précédemment gravides est de 13 au premier cestrus, 32 après les 2 premiers et seulement $55 \mathrm{p}$. Ioo après les 
4 premiers. Cette faible réussite résulte des anomalies dans le cycle sexuel qui s'établit dans les 45 jours post-partum, comme nous l'avons montré par ailleurs (TCHAMI'CHIAN et al., 1973).

Groupe 2 : La fertilité est très bonne aux luttes 2 et 4 de saison, mais elle est seulement de 3,3 p. Ioo à la lutte 3 de mai I970, ce qui confirme largement les résultats obtenus avec les brebis du groupe I à la même époque (tabl. I).

\section{TABLEAU 2}

Taux de mise bas des brebis lors des luttes 2 et 4 (groupe I)

\begin{tabular}{|c|c|c|c|c|c|c|}
\hline \multirow{2}{*}{$\begin{array}{l}\text { Numéro } \\
\text { de lutte }\end{array}$} & \multirow{2}{*}{ Catégorie } & \multirow{2}{*}{ Effectif } & \multicolumn{4}{|c|}{ Pourcentage de brebis gravides après } \\
\hline & & & 1er $^{\mathrm{er}}$ ostrus & $2^{\mathrm{e}}$ œstrus & $3^{\mathrm{e}}$ œstrus & $4^{e}$ ostrus \\
\hline \multirow{2}{*}{2} & $\mathrm{G}$ & 47 & 12,7 & 31,9 & 53,1 & 55,2 \\
\hline & $\mathrm{V}$ & 16 & 87,5 & 93,8 & - & - \\
\hline \multirow{2}{*}{4} & G & 4 & 0 & 0 & 0 & 0 \\
\hline & $\mathrm{V}$ & 53 & 83,0 & 92,5 & 94,2 & - \\
\hline
\end{tabular}

$\mathrm{G}=$ Gravides à la lutte précédente.

$\mathrm{V}=$ Vides (ou non gravides) à la lutte précédente.

\section{B. - Fertilité globale, prolificité et fécondité}

Globalement, la fertilité est de 75,8-65,I-7,o et 87,7 p. roo après les luttes I à 4 respectivement (tabl. 3). Le taux de prolificité varie également avec la saison : il est de 256 et 225 pour les mises bas consécutives aux luttes I et 3 qui ont lieu à contre-

TABLEAU 3

Fertilité, prolificité et fécondité des 4 luttes en 2 ans

(groupe I)

\begin{tabular}{|c|c|c|c|c|c|c|}
\hline \multirow{2}{*}{$\begin{array}{l}\text { Numéro } \\
\text { de lutte }\end{array}$} & \multicolumn{2}{|c|}{ Effectif } & \multirow{2}{*}{ Fertilité $(\%)$} & \multirow{2}{*}{ Prolificité $(\%)$} & \multicolumn{2}{|c|}{ Fécondité (\%) } \\
\hline & mis en lutte & mettant bas & & & par lutte & par année \\
\hline 1 & 66 & 50 & 75,8 & 256 & 194 & \\
\hline 2 & 63 & 41 & 65,1 & 293 & 190 & \\
\hline 3 & 57 & 4 & 7,0 & 225 & 16 & \\
\hline 4 & 57 & 50 & 87,7 & 340 & 298 & \\
\hline
\end{tabular}


saison, de 293 et 340 pour les mises bas consécutives aux luttes 2 et 4 qui ont lieu en saison sexuelle.

Pour avoir une idée de la productivité de ce schéma de reproduction, nous avons calculé la fécondité à chaque lutte et la fécondité annuelle : celle-ci est de $384 \mathrm{p}$. Ioo la première année (luttes I et 2) et de $3 \mathrm{I} 4 \mathrm{p}$. Ioo la deuxième année (luttes 3 et 4 ). Ces valeurs sont, dans l'ensemble, supérieures à la fécondité que l'on peut attendre d'une seule lutte annuelle en saison.

\section{DISCUSSION}

I. La comparaison des périodes de lutte et de mise bas des brebis du groupe I montre que, pour les 55 adultes, la première lutte de mai-juin r 969 commence 4 mois après le début des mises bas précédentes, alors que la troisième lutte de mai-juin r970 arrive seulement 2 mois après : la différence de réussite entre les luttes I et 3 qui ont lieu à la même date, peut donc s'expliquer par une interférence de l'anœstrus saisonnier et de l'anœstrus post-partum. Cependant, comme la fertilité a été particulièrement faible lors de la lutte 3 sur les 2 I brebis précédemment vides (4 seulement ont été fécondées), on peut supposer que l'activité sexuelle des brebis a été plus faible en mai I970 par rapport à celle de l'année précédente. Des variations annuelles dans la fréquence du comportement d'œestrus à cette époque ont d'ailleurs été signalées par THIMONIER et MAUI,ÉON (I969) et par LAND (I97I) : ainsi, ce dernier enregistre par exemple $59 \mathrm{p}$. Ioo de venues en ostrus au printemps de I968 avec I23 brebis Finnois $\times$ Dorset, contre 23 p. Ioo à la même époque, 1'année suivante, sur les I33 brebis présentes et par ailleurs plus âgées.

2. - Si la lutte de mai donne des résultats variables, mais dans tous les cas nettement inférieurs en fertilité et prolificité (cf. WHITEMAN et al., I972), la lutte en saison sexuelle est également moins fertile lorsqu'elle a lieu dans les 45 jours postpartum : 26 brebis fécondées sur $5 \mathrm{I}$ brebis mises en lutte pour l'ensemble des luttes 2 et 4 , soit une fertilité de 5r,o p. Ioo contre 94,2 pour les 69 brebis correspondantes ayant mis bas depuis au moins 7 mois.

Dans leur expérience de rythme intensif de reproduction ( 4 mises bas en 2 ans) sur des croisées Finnois $\times$ Dorset, LAND et MCCLEL,AND (I97I) montrent également que les mises bas trop rapprochées réduisent nettement le taux de fertilité : pour les 2 dernières luttes, ce taux est de $9 \mathrm{I}$ pour les brebis non fécondées à la lutte précédente, contre 32 pour les brebis gravides. Même en utilisant 1'insémination artificielle après synchronisation des chaleurs, sur les brebis croisées Romanov $\times$ Limousine, THÉRIEZ (I972) constate une fertilité assez faible en avril pour les brebis ayant mis bas précédemment en mars.

3. - Sur les 55 brebis du groupe I, ayant mis bas fin I968-début 1969,24 seulement ont été fécondées au cours des luttes $\mathrm{I}$ et 2 , mais aucune n'a reproduit 4 fois de suite. Le résultat est encore moins favorable pour les 3 I agnelles introduites à la deuxième lutte. Le système des 2 luttes annuelles en mai et octobre utilisé à Bourges sur les brebis Romanov, ou celui pratiqué par LAND et MCCLELLAND (I97I) en mars-avril et août-septembre sur brebis croisées Finnois $\times$ Dorset, apparaît 
finalement peu efficace. Pour obtenir de meilleurs résultats, il semble nécessaire de prévoir atı moins 3 périodes annuelles de lutte ou d'essayer d'utiliser la longue saison sexuelle des brebis Romanov. LAND et al. (I973) ont en effet montré que l'activité sexuelle de ces brebis en contrôle permanent d'œstrus était maximum de septembre à décembre-janvier, mais se prolongeait largement jusqu'à la fin avril, début mai pour certains animaux. En effectuant des saillies en juillet-août (au tout début de la saison sexuelle) et en janvier-février (c'est-à-dire avant la fin de la saison sexuelle) il doit être possible d'obtenir de bons résultats en fertilité, avec vraisemblablement une prolificité réduite par rapport à celle obtenue en pleine saison. Dans sa revue bibliographique, Desvignes (r97I) montre que le double agnelage a été pratiqué de cette façon, en Russie, sur des effectifs élevés, certains élevages réalisant couramment cette performance sur 30 à 40 p. Ioo de leurs effectifs. Il s'agit là d'un résultat qu'il importe de vérifier sur plusieurs années consécutives, pour éprouver les possibilités d'utilisation intensive de cette race et, si possible, déceler les souches qui répondent le mieux à un tel système.

\title{
Reçu pour publication en avril 1973.
}

\section{REMERCIEMENTS}

Nous remercions MM. Mauléon et Thimonier (Station de Recherches sur la Physiologie de la Reproduction, C. R. de Tours) et M. ThéRIEz (Station de Recherches sur l'Élevage des Ruminants, C. R. V. Z. de Theix), des critiques et suggestions qu'ils nous ont apportées à la lecture de ce mémoire.

\section{SUMMARY}

\author{
PERFORMANCES OF ROMANOV EWES SUBJECTED
}

TO AN ACCEIERATED RHYTHM OF REPRODUCTION

The system of 4 matings in 2 years (Ist mating in may-june I969, 2nd mating from september

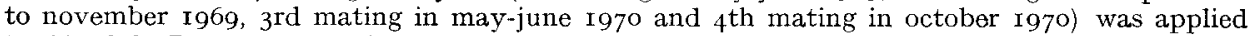
to 66 adult Romanov ewes (group $\mathrm{r}$ among which 55 had lambed 3 to 4 months before the Ist mating (tabl. $\mathbf{I}$ ).

Overall conception rates after $\mathrm{Ist}, 2 \mathrm{nd}, 3 \mathrm{rd}$ and 4 th mating were $75.8 ; 65 . \mathrm{I} ; 7.0$ and 87.7 p. Ioo, respectively; the corresponding figures for prolificacy were $256,293,225$ and 340 p. Ioo (tabl. 3 ). When considering only the ewes pregnant at the previous mating, the conception rate (p. roo) was 87,3 after the Ist mating, 55.3 after the second one and 0 after matings 3 and 4 (tabl. I).

The results obtained with 3 I ewe lambs (group LL) mated for the first time at the 2 nd mating in september-october 1969 confirm the poor results obtained after the 3 rd mating in may $197^{\circ}$ (tabl. I).

To obtain higher productivity in ewes subjected to an accelerated rhythm of reproduction changes in the periods of mating seem to be necessary with a view to taking better into account the sexual activity of Romanov ewes according to the season.

\section{RÉFÉRENCES BIBLIOGRAPHIQUES}

Desvignes A.,I97I. La race Romanov. Revue bibliographique. Ann. Zootech. 20, 353-37o.

LAND R. B., I97I. The incidence of ostrus during lactation in Finnish Landrace, Dorset Horn and Finn-Dorset sheep. J. Reprod. Fert., 24, 345-352. 
LANd R. B., MCCLElland T. H., I97I. The performance of Finn-Dorset sheep allowed to mate four times in two years. Anim. Prod., 13, 637-64I.

Land R. B., Pelletier J., Thimonier J., Mauléon P., 1973. J. Endocr. (in press).

Tchamitchian L., Ricordeau G., Lefìve C., Desvignes A., I973. Observations sur l'anostrus post-partum des brebis Romanov après un agnelage en saison sexuelle. Ann. Zootech. 22, 295-30I.

Thimonier J., Mauléon P., I969. Variations saisonnières du comportement d'oestrus et des activités ovarienne et hypophysaire chez les ovins. Ann. Biol. anim. Bioch. Biophys., 9, 223-250.

ThÉRIEZ, 1972. Communication personnelle.

Whiteman J. V., Zollinger W. A., Thrift F. A., Gould M. B., I972. Post partum mating performance of ewes involved in a twice-yearly lambing program. J. Anim. Sci., 35, 836-842. 